\title{
An investigation of intrinsic gravity wave signatures using coordinated lidar and nightglow image measurements
}

\author{
M.J. Taylor \\ Space Dynamics Laboratory and Physics Department, Utah State University \\ Y.Y. Gu, X. Tao, C.S. Gardner \\ Department of Electrical and Computer Engineering, University of Ilinois at Urbana-Champaign
}

M.B. Bishop

Space Dynamics Laboratory and Physics Department, Utah State University

\begin{abstract}
Simultaneous observations of gravity waves using an $\mathrm{Na}$ wind/temperature lidar and a multi-wavelength all-sky nightglow imager were obtained, for the first time, during the ALOHA-93 campaign. A novel investigation of intrinsic wave parameters has been made by combining measurements of the horizontal wave components imaged in four nightglow emissions (height range $\sim 80-100 \mathrm{~km}$ ) with $\mathrm{Na}$ lidar soundings of the horizontal wind field and temperature profiles over the same height interval. On October 19 both instruments registered marked monochromatic wave motions. The intrinsic periods of several of these waves have been determined and were found to vary considerably with altitude, often resulting in a significant increase over their observed wave periods. It is shown that these two instrumental techniques generally sampled different regions of the gravity wave spectrum: the lidar exhibiting most sensitivity to short vertical wavelength waves (less than about $10 \mathrm{~km}$ ) while the imager was most responsive to larger vertical wavelength waves. This study illustrates the significant advantages of combining wind/temperature lidar and multi-wavelength image observations for intrinsic gravity wave measurements.
\end{abstract}

\section{Introduction}

In recent years several powerful resonant lidar systems have been developed that are capable of sounding the vertical properties of gravity waves propagating through the upper mesosphere and lower thermosphere $(80-100 \mathrm{~km})$ in exceptional detail [e.g. Beatty et al., 1992; She et al., 1992]. New CCD imaging systems have also been developed permitting accurate measurements of the horizontal wave parameters using visible and near infrared (NIR) nightglow emissions [e.g. Taylor et al., 1995]. On their own, each of these instruments has been successful at measuring some, but usually not all, of the parameters needed to characterize the intrinsic wave motions [e.g. Gardner and Voelz, 1987; Taylor et al., 1987]. Intrinsic measurements are important as they describe the true motion of the gravity wave: its period, speed and angle of ascent/descent through the atmosphere which are necessary to investigate wave coupling and momentum deposition in the vicinity of the mesopause. By combining simultaneous lidar and image measurements of the vertical and

Copyright 1995 by the American Geophysical Union.

Paper number 95GL02949

0094-8534/95/95GL-02949\$03.00 horizontal wave motions, it is hoped to determine the intrinsic properties of many types of gravity waves. The underlying assumption being that both instruments sample the same part of the gravity wave spectrum. However, measurements of intrinsic wave parameters are currently very rare.

During the ALOHA-93 campaign coincident $\mathrm{Na}$ lidar and image measurements were made on a number of occasions, from both the NCAR aircraft and from the ground. In particular, the ground base lidar system was capable of measuring winds and temperatures at the same time as the wave measurements. In this letter we perform a unique study using simultaneous $\mathrm{Na}$ wind/temperature (W/T) lidar data (University of Illinois) and multi-wavelength CCD imager data (Utah State University) to investigate the intrinsic properties of several wave motions and, at the same time, assess the spectral range and sensitivities of these two systems. Two intervals on 19 October have been selected for this study when marked wave structure was detected by both instruments.

\section{Operational Details}

The Na W/T lidar was installed at the Air Force Maui Optical Site (AMOS) located on the summit of Haleakala Crater, Maui $\left(20.8^{\circ} \mathrm{N}, 156.2^{\circ} \mathrm{W}, 3050 \mathrm{~m}\right)$. The laser was interfaced with a $0.8 \mathrm{~m}$ diameter Beam Director Telescope providing full scanning capabilities for the lidar system. To measure all three wind components and atmospheric temperature the lidar was pointed sequentially at zenith and $15^{\circ}$ off zenith to the $\mathbf{N}$ and $E$. The cycle time for one complete set of measurements was $\sim 15$ min. The wind and temperature data were smoothed during analysis yielding profiles with $30 \mathrm{~min}$ and $1 \mathrm{~km}$ resolution. The absolute accuracy of the measurements, throughout the Na layer (typically $85-100 \mathrm{~km}$ ), was better than 5 $\mathrm{ms}^{-1}$ for the horizontal winds and $2 \mathrm{~K}$ for temperature. The $\mathrm{Na}$ density profiles were obtained at resolutions of $5 \mathrm{~min}$ and $48 \mathrm{~m}$.

The all-sky imager was located $\mathbf{2 0 0} \mathrm{m}$ from the lidar at the DOE Facility. The imager was used to measure wave structure over a very large geographic area $\left(>6 \times 10^{5} \mathrm{~km}^{2}\right)$ and over a similar altitude range as the lidar measurements $(-80-100 \mathrm{~km})$ [Taylor et al., 1995]. This was achieved by sampling sequentially four nightglow emissions that peak in intensity at different heights: the NIR $(715-930 \mathrm{~nm}) \mathrm{OH}$ bands at $\sim 87 \mathrm{~km}$, the $\mathrm{O}_{2}(0,1)$ At band at $\sim 94 \mathrm{~km}$, the OI(557.7 $\mathrm{nm})$ line at $\sim 96 \mathrm{~km}$ and the $\mathrm{Na}(589.2 \mathrm{~nm})$ lines at $\sim 90 \mathrm{~km}$. Integration times ranged from 20 s for the bright $\mathrm{OH}$ bands to 120 s for the faint $\mathrm{Na}$ lines resulting in a cycle time of $\rightarrow 9 \mathrm{~min}$. Coordinated lidar and image 

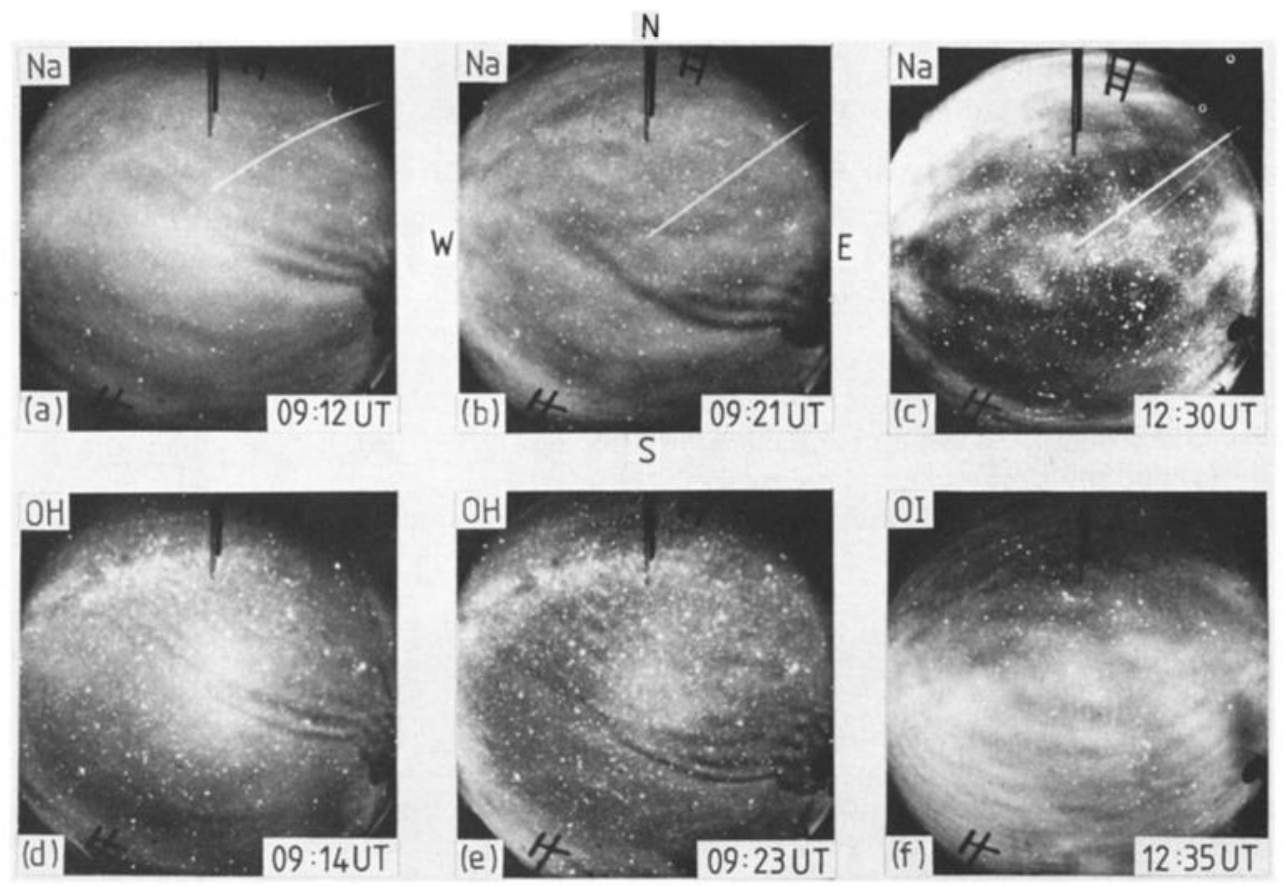

Figure 1. All-sky CCD data illustrating the variety of wave structure recorded on the 19 October. Images $a, b, d, e$ show examples of the wave packet imaged in the $\mathrm{Na}$ and $\mathrm{OH}$ emissions during the first interval (09:0010:00 UT). Images $c, f$ were recorded during the second interval (12:00-13:30 UT) and show the small-scale ripples imaged in the $\mathrm{Na}$ emission at 12:30 UT, and the E-W band structure present in the OI $(557.7 \mathrm{~nm})$ emission at 12:35 UT. Note the faint bands to the NW in both images and the absence of ripples in OI data.

measurements were made from 6-23 October and a variety of wave motions were measured.

\section{Observations and Results}

On 19 October coincident lidar and image measurements were made from $06: 45$ to $13: 25 \mathrm{UT}$ from which two periods have been selected for analysis: 09:00-10:00 UT and 12:00-13:30 UT.

During the first interval a group of 3-4 coherent "bands" was imaged in all four nightglow emissions. Figure 1a,b shows two examples of these bands imaged in the Na emission at 09:12 UT and 09:21 UT illustrating their passage through the lidar sample volume. For comparison, two $\mathrm{OH}$ images (Figure 1d,e) are presented to show this wave packet at a different emission altitude. The limited number of wave crests comprising this display was most unusual as was the distinctive kink in the wave field (to the E) which was present in each of the emissions. This event was detected first at $\sim 08: 30 \mathrm{UT}$ when it was well to the NNE of Maui. The waves exhibited rapid motion, passing overhead at $\sim 09: 21$ UT (Figure 1b). Table 1 lists the wave parameters determined from the image data. Within the limits of the measurements all four emissions exhibited the same horizontal wavelength $\left(\lambda_{4}\right)$ of $14.3 \pm 0.7 \mathrm{~km}$, and a horizontal phase speed $\left(v_{b}\right)$ of $60 \pm 2 \mathrm{~ms}^{-1}$ indicating an observed wave period ( $T_{\text {obr }}$ ) of only $4.0 \pm 0.2 \mathrm{~min}$ (close to the local Brunt-Väisălā period). For comparison Figure 2 shows a sequence of $\mathrm{Na}$ density profiles for the same period. The $\mathrm{Na}$ layer was clearly perturbed by a wave-like motion exhibiting a $-6 \%$ amplitude variation in relative density and a vertical wavelength $(\lambda z)$ of $\sim 5.5 \mathrm{~km}$. The profile sequence also shows a downward phase progression of $-0.5 \mathrm{~ms}^{-1}$ yielding a much longer observed period of $\sim 183 \mathrm{~min}$ for these waves.

During the second interval (12:00-13:30 UT) a more complex situation existed (as depicted by the image data) consisting of a group of small-scale "ripple" waves and two sets of "bands". Ripples occur over limited areas of sky and exhibit short lifetimes $<45$ min [Taylor and Hapgood, 1990]. On this occasion the ripples were most prominent in the Na emission (Figure 1c). Close inspection of this figure reveals over 20 wave crests with $\lambda_{h}$ of $5.2 \mathrm{~km}$. This display was detected first at low elevations

Table 1. Measurements of the horizontal wave parameters determined from the image data and the derived intrinsic wave periods, buoyancy periods and vertical wavelengths using the lidar data.

\begin{tabular}{|c|c|c|c|c|c|c|c|c|c|c|}
\hline Emission & $\begin{array}{c}\text { Height } \\
(\mathrm{km})\end{array}$ & $\begin{array}{l}\text { Time } \\
\text { (UT) }\end{array}$ & $\begin{array}{c}\text { Azimuth } \\
\left.\text { ( }{ }^{\circ} \mathrm{N}\right)\end{array}$ & $\begin{array}{c}\lambda_{h} \\
(\mathbf{k m})\end{array}$ & $\begin{array}{c}\mathbf{V h} \\
\left(\mathbf{m s}^{-1}\right)\end{array}$ & $\begin{array}{c}\mathrm{T}_{\text {obs }} \\
\text { (min) }\end{array}$ & $\begin{array}{c}T_{\mathbf{b}} \\
(\mathrm{min})\end{array}$ & $\begin{array}{c}\mathrm{U} \\
\left(\mathrm{ms}^{-1}\right)\end{array}$ & $\begin{array}{c}\mathbf{T}_{\mathbf{i}} \\
(\min )\end{array}$ & $\begin{array}{r}\lambda_{z} \\
(\mathrm{~km})\end{array}$ \\
\hline OH & 87 & 09:00-09:40 & 200 & $13.2 \pm 0.7$ & $58.3 \pm 0.4$ & $3.8 \pm 0.2$ & 5.0 & 13 & 4.9 & - \\
\hline $\mathrm{Na}$ & 90 & 09:00-09:40 & 200 & $14.4 \pm 0.5$ & $58.5 \pm 0.7$ & $4.1 \pm 0.2$ & 5.0 & 29 & 8.1 & 11 \\
\hline $\mathrm{O}_{2}$ & 94 & 09:00-09:40 & 200 & $14.9 \pm 0.7$ & $62.3 \pm 0.9$ & $4.0 \pm 0.2$ & 4.8 & 29 & 7.5 & 12 \\
\hline OI & 96 & 09:00-09:40 & 200 & $14.6 \pm 0.4$ & $60.9 \pm 0.6$ & $4.0 \pm 0.1$ & 4.8 & 30 & 7.9 & 11 \\
\hline $\mathbf{N a}$ & 90 & $12: 00-12: 45$ & $\sim 0$ & $5.2 \pm 0.1$ & - & - & 5.1 & - & - & - \\
\hline OI & 96 & $12: 00-12: 45$ & 190 & $21 \pm 2$ & $42 \pm 3$ & $8.3 \pm 1.0$ & 5.2 & -18 & 5.8 & 41 \\
\hline $\mathbf{N a}$ & 90 & $12: 00-12: 45$ & 240 & $30 \pm 5$ & $38 \pm 3$ & $13.2 \pm 2.4$ & 5.1 & 3.4 & 17.6 & 11 \\
\hline
\end{tabular}




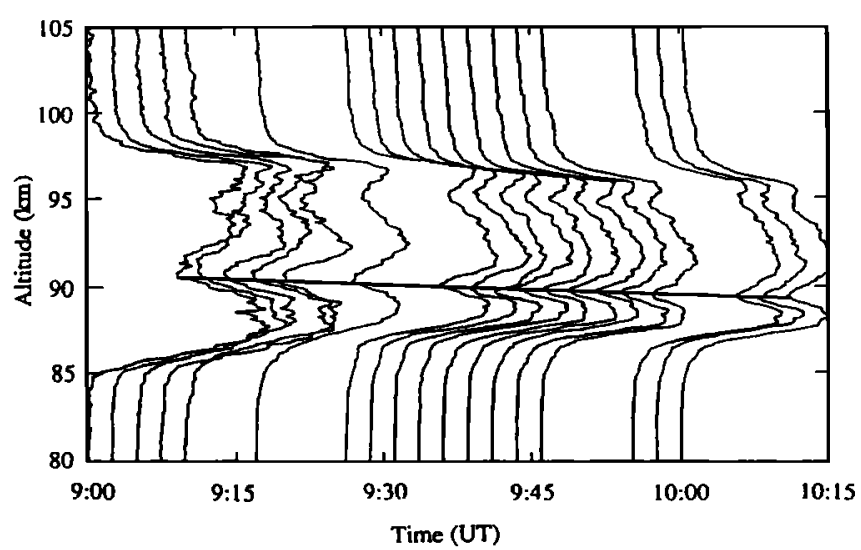

Figure 2. Na density profiles recorded during the first interval. The Na layer was perturbed by a gravity wave of $\lambda_{z} \sim 5.5 \mathrm{~km}$ exhibiting a downward vertical phase speed of $\sim 0.5 \mathrm{~ms}^{-1}$.

to the $S$ around 12:00 UT and was observed to grow rapidly towards the N. By 12:30 UT the ripples were close to the zenith and extended to the $\mathrm{E}$ and $\mathrm{W}$ of Maui (Figure 1c). However, in the next $\mathrm{Na}$ image ( $\sim 9$ min later) the ripples had faded considerably and it is not clear whether these waves passed through the lidar sample volume. Similar morphology, but much fainter, ripples were also detected in the $\mathrm{OH}$ and $\mathrm{O}_{2}$ emissions but not in the OI emission indicating that they occurred over a restricted height range centered near $\sim 90 \mathrm{~km}$. Velocity measurements of this display were not possible due to its rapid evolution.

Figure 1f shows the wave structure present in OI emission around this time (12:35 UT). Several near E-W aligned bands are seen filling the southern sky (where the ripples occurred at lower heights). These bands were observed to propagate in the same general direction (azimuth $\sim 190^{\circ} \mathrm{N}$ ) as the bands observed earlier this night, but they exhibited a longer wavelength, $\lambda_{h} \sim 21$ $\mathrm{km}$ and a lower phase speed, $\mathrm{v}_{\mathrm{h}} \sim \mathbf{4 2} \mathrm{ms}^{-1}$ indicating a larger $T_{\text {obs }}$ 8.3 min (Table 1). Also evident in both the $\mathrm{Na}$ and OI images of Figure 1c,f are some very faint bands in the NW sky progressing towards the SSW (azimuth $-240^{\circ} \mathrm{N}$ ). Measurements of these waves yield a $\lambda_{\mathrm{h}} \sim 30 \mathrm{~km}$ and a $\mathrm{vh}_{\mathrm{h}} \sim 38 \mathrm{~ms}^{-1}$ indicating a $\mathrm{T}_{\text {obs }} \sim 13.2 \mathrm{~min}$. Figure 3 shows a sequence of $\mathrm{Na}$ density profiles for this period. The Na layer broadened considerably towards the end of this interval and downward propagating waves (of relative density amplitude $\sim 2.8 \%$ ) exhibiting a $\lambda_{2}$ $-3.7 \mathrm{~km}$ and vertical phase speed of $\sim 1 \mathrm{~ms}^{-1}$ are evident in all the profiles indicating an observed wave period of $-62 \mathrm{~min}$.

\section{Discussion}

On numerous occasions during this campaign it was noted that the occurrence of wave activity in the lidar data often coincided with prominent wave structure in the image data. This situation prevailed on 19 October when an abundance of quasi-monochromatic waves were detected. A qualitative assessment of the data obtained during the first interval suggests that the coherent wave packet imaged in all four nightglow emissions was associated with the detection by lidar of marked wave structure over the full height range of the $\mathrm{Na}$ density profile $(\sim 85-100 \mathrm{~km})$. Similarly, during the second interval when ripples were imaged in the $\mathrm{Na}$ emission, the lidar indicated small amplitude variations around the $\boldsymbol{\sim 9 0} \mathbf{~ k m}$ level. The lidar data also revealed a wave motion $\left(\lambda_{z} \sim 3.7 \mathrm{~km}\right)$ which exhibited its largest amplitude variation near top of the $\mathrm{Na}$ density layer at $\sim 95 \mathrm{~km}$ in general agreement with the detection of strong band structure in the OI emission. In each case the difference in the observed periodicities of the wave motions as measured by both instruments may be attributed to the effects of background winds on the vertical phase progression. Taken together these observations suggest that both instruments sampled the same gravity wave disturbances and that a combination of the horizontal and vertical wave data may therefore be used to determine their intrinsic parameters. However, this comparison is overly simplistic and in order to quantify the intrinsic properties of the gravity waves at any given time the wind and temperature measurements provided by the W/T lidar are needed to establish the prevailing atmospheric conditions.

In the presence of a background wind the intrinsic period $\left(T_{i}\right)$ of the wave motion is Doppler shifted with respect to its observed period:

$$
T_{i}=T_{\text {obs }}\left(v_{h} /\left(v_{h}-U\right)\right)
$$

where $\mathrm{U}$ is the horizontal background wind in the direction of the wave. Figure 4 plots the meridional (u) and zonal (v) wind profiles, temperature profile $(T)$ and the buoyancy period profile $\left(T_{b}\right)$ determined from the lidar data for the first observation period. (Similar profiles for the second interval are given in Figure 5.) During the first interval the background winds over the entire height range of the nightglow layers (Figure 4a) were directed in the same sense as that of the wave motion indicating that the intrinsic wave periods for each emission were higher than their observed values. Calculations for $T_{i}$ and $\lambda_{z}$ are given in Table 1 for each nightglow emission together with the mean values of $U$ and $T_{b}$ determined by averaging wind and temperature data over $\sim 4 \mathrm{~km}$ height range centered on each layer (except the $\mathrm{OH}$ layer for which there are no data below $\sim 87 \mathrm{~km}$ ). It is evident that during this time the instantaneous value of $T_{b}$ varied significantly with height, probably due to changes in the temperature gradient (Figure 4b) induced by the wave perturbations. However, as these changes do not reflect the background state of the atmosphere, $\mathbf{T}_{\mathbf{b}}$ was computed using a mean temperature for each emission layer and assuming a zero temperature gradient. Values for the intrinsic wave period varied from $\sim 5 \mathrm{~min}$ at the $\mathrm{OH}$ level to $\sim 8 \mathrm{~min}$ at higher altitudes indicating a significant increase over their observed period (Table 1). The vertical wavelengths were calculated using the Boussinesq approximation for the gravity wave dispersion relations and the determined values of $\lambda_{b}, T_{i}$ and $T_{b}$. In each case the value for $\lambda_{z}$ was found to be $\sim 11 \mathrm{~km}$ indicating a vertical scale that is comparable to the full depth of the $\mathrm{Na}$

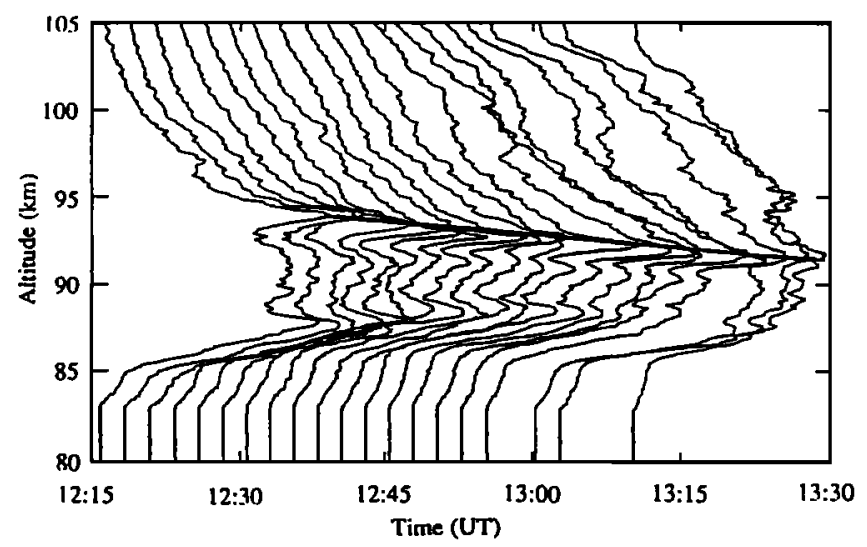

Figure 3. $\mathrm{Na}$ density profiles measured during the second interval. The Na layer was perturbed by a gravity wave with a $\lambda_{z} \sim 3.7 \mathrm{~km}$ and a downward vertical phase speed of $\sim 1.0 \mathrm{~ms}^{-1}$. 

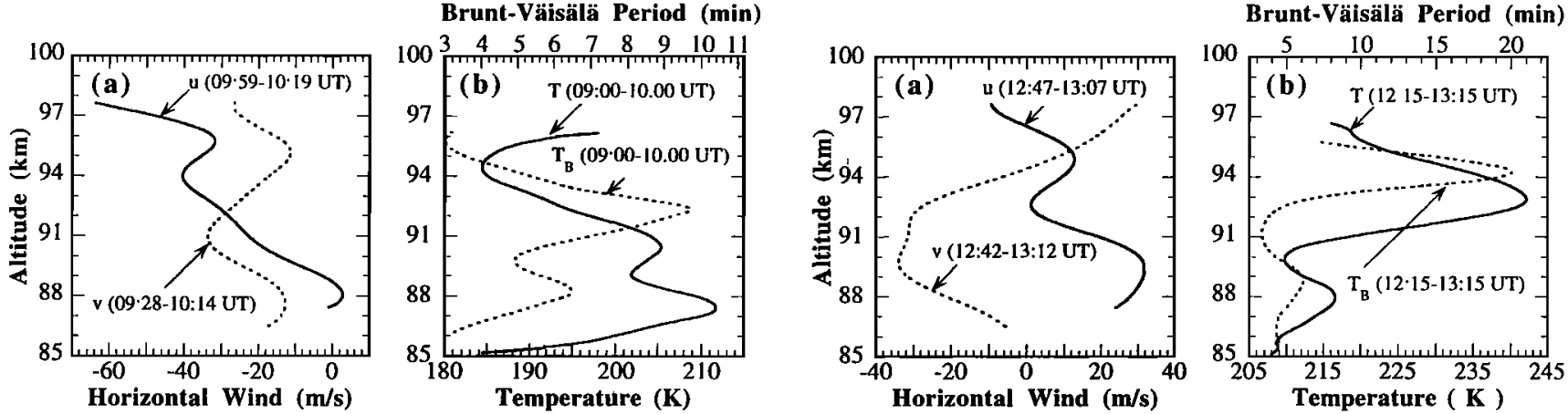

Figure 4. (a) Average meridional ' $u$ ' and zonal ' $v$ ' wind profiles and (b) average atmospheric temperature ' $T$ ' and buoyancy period ' $\mathrm{T}_{\mathbf{b}}$ ' profiles measured by the lidar during the first interval.

density layer and would therefore not be detected readily in the lidar data. In contrast, the long period ( $183 \mathrm{~min})$ waves measured by the lidar indicate a $\lambda_{h} \sim 200 \mathrm{~km}$ for the same prevailing conditions. As this corresponds to a large fraction of the all-sky field ( $-2 / 3$ image centered on zenith) this wave motion would also be difficult to detect by visual inspection of the image data. Thus the situation for the first interval (09:0010:00 UT) indicates that the wave forms detected in each data set are apparently not orthogonal projections of the same gravity wave motion. This result is important, but not surprising in this case, as the lidar data clearly show downward phase progression indicative of a freely propagating wave while the airglow data indicate short period waves with no phase variation with altitude (not shown) suggesting a ducted or evanescent wave motion.

For the second interval (12:00-13:30 UT) the zonal winds (Figure 5a) were directed eastward (opposite to the earlier period) and ranged from $\sim 0$ to $30 \mathrm{~ms}^{-1}$ peaking at $\mathrm{Na}$ heights. The meridional winds exhibited a significant shear of $\sim 55 \mathrm{~ms}^{-1}$ between $\mathrm{Na}$ and $\mathrm{OI}$ heights. Wind shears are thought to be the primary source of ripples (through the Kelvin-Helmholtz instability), and their prominence in the $\mathrm{Na}$ emission during this period is consistent with this hypothesis. However, the magnitude of the shear appears to be insufficient to account for the observed structures [Taylor and Hapgood, 1990].

The headings of the two band-type motions imaged during the second interval differed by $\sim 50^{\circ}$. Consequently the effects of the background winds were quite different; significantly reducing the intrinsic period of the S-ward propagating wave to $\sim 5.8$ min, whilst having almost no effect on the wave motion progressing towards the WSW as the winds were near orthogonal to the wave heading (Table 1). In both cases the vertical wavelengths were again found to be similar to, or larger than, the $\mathrm{Na}$ density layer profile and so were not the origin of the $\lambda_{z} \sim 3.7$ $\mathrm{km}$ wave motion observed by the lidar. Further, more detailed, analysis of these data is required to identify the signatures of these wave events in each data set.

\section{Summary}

The novel combination of wind/temperature lidar soundings with multi-wavelength image measurements provides a powerful technique for detailed studies of gravity wave propagation in the $\sim 80-100 \mathrm{~km}$ height region. Initial analysis of this joint data set has yielded new information on the intrinsic parameters of several short period wave motions and paves the way for an indepth investigation of wave propagation and dissipation over the mid-Pacific ocean. It is clear, even from this limited study, that the spectrum of waves to which each instrument is most sensitive is controlled by layer thickness; the lidar being most sensitive to short $\lambda_{z}$ waves, less than the width of the Na density profile $(\sim 10 \mathrm{~km})$, while the imager is most sensitive to distur-

Figure 5. (a) Average meridional ' $u$ ' and zonal ' $v$ ' wind profiles and (b) average atmospheric temperature ' $T$ ' and buoyancy period ' $\mathrm{T}_{\mathrm{b}}$ ' profiles for the second interval.

bances of $\lambda_{z}$ greater than the airglow layer widths (i.e. typically $>10 \mathrm{~km}$ ). Together these observations offer the capability to cover a much larger spectral range than is possible individually and hence to enhance our capability for intrinsic wave measurements. A significant region of spectral overlap also exists where the horizontal and vertical signatures of many gravity waves should be identifiable in each data set. For these cases the data are over sampled and the unique combination of wind, temperature and wave measurements provided by these two instruments may be used to investigate the wave properties (i.e. freely propagating or ducted) in unprecedented detail.

Acknowledgments. We are most grateful to J.A. Albetski, Thermo Trex Corp., for arranging our use of the AMOS and DOE Facilities. The Illinois group was supported in part by National Science Foundation (NSF) grants ATM-24367 and ATM94-03036. The Utah State Univensity measurements were supported by an NSF CEDAR grant ATM-9302844. Partial support for the image analysis was provided by the Geophysics Directorate, Air Force Phillips Laboratory, contract No. F19628-93-C-0165 under the SOAR program.

\section{References}

Beatty, T. J., C. A. Hostetler, and C. S. Gardner, Lidar observations of gravity waves and their spectra near the mesopause and stratopause at Arecibo, J. Atmos. Sci., 49, 477-496, 1992.

Gardner, C. S., and D. G. Voelz, Lidar studies of the nighttime sodium layer over Urbana, Illinois, 2, Gravity waves, J. Geophys. Res., 92, 4673-4694, 1987.

She, C. Y., J. R. Yu, H. Latifi, and R. E. Bills, High spectral resolution lidar for mesospheric sodium temperature measurements, Appl. Opt., 31, 2095-2106, 1992.

Taylor, M.J., M. B. Bishop, and V. Taylor, All-sky measurements of short period waves imaged in the OI(557.7 nm), $\mathrm{Na}(589.2 \mathrm{~nm})$ and near infrared $\mathrm{OH}$ and $\mathrm{O}_{2}(0,1)$ nightglow emissions during the ALOHA-93 campaign, Geophys. Res. Lett., this issue, 1995.

Taylor, M.J., and M.A. Hapgood, On the origin of ripple-type wave structure in the $\mathrm{OH}$ nightglow emission, Planet. Space Sci., 38, 1421-1430, 1990.

Taylor, M.J., M.A. Hapgood, and P. Rothwell, Observations of gravity wave propagation in the OI $(557.7 \mathrm{~nm}), \mathrm{Na}(589.2 \mathrm{~nm})$ and the near infrared $\mathrm{OH}$ nightglow emission, Planet. Space Sci., 35, 413 427, 1987.

M.J. Taylor, and M.B. Bishop, Space Dynamics Laboratory, Utah State University, Logan, UT 84322-4145 (e-mail: Taylor@zeus.sdl. usu.edu).

Y.Y. Gu, X. Tao, and C.S. Gardner, Department of Electrical and Computer Engineering, University of Illinois at Urbana-Champaign, 1308 West Main Street, Urbana, IL 61801 (e-mail: Yygui@ux1.cso. uiuc.edu; Cgardner@uiuc.edu).

(Received December 15, 1994; revised September 4, 1995; accepted September 11, 1995) 